\title{
A GLOBAL INVARIANT OF CONFORMAL MAPPINGS IN SPACE
}

\author{
JAMES H. WHITE
}

\begin{abstract}
This paper shows that the total integral of the square of the mean curvature for a compact orientable surface in $E^{3}$ is an invariant of a conformal space mapping. This result is then used to answer a problem raised by $\mathrm{T}$. Willmore and B.-Y. Chen concerning embeddings of compact orientable surfaces, and in particular tori, for which this integral is a minimum.
\end{abstract}

A conformal mapping on Euclidean three-space has the property that it carries spheres into spheres. Such a mapping can be decomposed into a product of similarity transformations and inversions. Let $x: M^{2} \rightarrow E^{3}$ be a $C^{3}$ regular immersion of a compact orientable surface into Euclidean three-space, and let $T=\int_{I^{2}} H^{2} d A$, where $H$ is the mean curvature of the immersed surface and $d A$ is the area element. This note proves the result that $T$ is invariant under a conformal space mapping and applies this result to the problem mentioned by T. J. Willmore [4], Shiohama and Takagi [3], and B.-Y. Chen [2], inter alia, of characterizing embeddings of the torus in three-space for which $\inf _{i} \int_{i(M)} H^{2} d A$ is achieved for all embeddings $i$.

That $T$ is invariant under similarity transformations (Euclidean motions and homotheties) is obvious. What is not so apparent is that $T$ is invariant under inversions. (We stipulate here that the center of inversion does not lie on the immersed surface.) It was observed locally by Blaschke [1] that the quantity $\left(H^{2}-K\right) d A$ is an inversion invariant, where $K$ is the Gauss curvature.

To prove this fact let the center of inversion be taken as the origin. Then, if $c$ is the radius of inversion, the position vector $\bar{x}$ of a point on the inverse surface, corresponding to the point $x$ on the original surface, has the direction of $x$ and the magnitude $c^{2} /|x|$ where $|x|$ denotes the length of $x$. Therefore, we may write $\bar{x}=c^{2} x /|x|^{2}$. If $N$ denotes the usual surface

Received by the editors June 15, 1972.

AMS (MOS) subject classifications (1970). Primary 53A05; Secondary 53A30, $53 \mathrm{C} 45$.

Key words and phrases. Conformal mappings in space, inversion, mean curvature, global invariant, anchor ring, torus.

1 The preparation of this paper was supported in part by NSF Grant GP-27576.

(c) American Mathematical Society 1973 
normal for the surface $x$, and if we set $h=x \cdot N$, a straightforward calculation yields

$$
\bar{k}_{1}=-\frac{|x|^{2}}{c^{2}} k_{1}-\frac{2 h}{c^{2}}, \quad \bar{k}_{2}=-\frac{|x|^{2}}{c^{2}} k_{2}-\frac{2 h}{c^{2}},
$$

where $\bar{k}_{i}$ and $k_{i}$ are the principal curvatures of the surface $\bar{x}$ and $x$ respectively. Hence, $\left(\bar{k}_{1}-\bar{k}_{2}\right)=-|x|^{2}\left(k_{1}-k_{2}\right) / c^{2}$, and this gives directly

$$
\bar{H}^{2}-\bar{K}=|x|^{4}\left(H^{2}-K\right) / c^{4}
$$

where $R, K$ and $\bar{H}, H$ denote the Gauss and mean curvatures of $\bar{x}$ and $x$. Another calculation shows that

$$
d \bar{A}=c^{4} d A /|x|^{4} .
$$

These last two results yield the statement of Blaschke:

$$
\left(\bar{H}^{2}-\bar{K}\right) d \bar{A}=\left(H^{2}-K\right) d A .
$$

The global result follows immediately, for if $\left(H^{2}-K\right) d A$ is an inversion invariant, $\int_{M^{2}}\left(H^{2}-K\right) d A$ certainly is. But

$$
\int_{M^{2}}\left(H^{2}-K\right) d A=\int_{M^{2}} H^{2} d A-2 \pi \chi\left(M^{2}\right),
$$

where $\chi\left(M^{2}\right)$ is the Euler characteristic. Hence, $\int_{M^{2}} H^{2} d A=T$ is an inversion invariant since $\chi\left(M^{2}\right)$ clearly is.

Applications. In [4], Willmore raised the question of finding the $\inf _{i} \int_{i(M)} H^{2} d A$ where $i$ ranges over all embeddings of an orientable compact surface $M^{2}$ in $E^{3}$. Our result shows the infimum is determined only up to conformal transformation. This observation is particularly noteworthy in the case of the torus. In [2], B.-Y. Chen shows as a special case of a more general theorem that an oriented compact surface in $E^{3}$ is stable with respect to the integral $\int_{M^{2}} H^{2} d A$, i.e. $\int_{M^{2}} H^{2} d A$ attains an extremal value with respect to normal variations, if and only if

$$
\Delta H+2 H^{3}-2 H K=0,
$$

where $\Delta$ denotes the Laplacian and $K$ the Gauss curvature. He observes that, for the case where $M^{2}$ is the torus, equation (1) is satisfied for the special standard tori called anchor rings whose generating circles have radii in the ratio $1: \sqrt{ } 2$. In this case $\int_{M^{2}} H^{2} d A=2 \pi^{2}$ is the infimum for all anchor rings, a fact first shown by Willmore. He then raises the question hinted at by both Willmore [4] and Shiohama-Takagi [3] as to whether these special anchor rings are the only unknotted tori in $E^{3}$ which satisfy equation (1). Our result can be extended to give a negative answer to this question. It is not difficult to see that if $\int_{M^{2}} H^{2} d A$ is an extremal 
value for a surface, it is also an extremal value for the inverse surface. (One can calculate and show directly that equation (1) is a conformal invariant.) Hence, any inversion of the anchor rings mentioned above is stable with respect to the integral $\int_{M^{2}} H^{2} d A$. These inversions are among the special class of tori called the cyclides of Dupin, tori that have the property that their lines of curvature are circles.

\section{REFERENCES}

1. W. Blaschke, Vorlesungen über Differentialgeometrie. III, Springer, Berlin, 1929.

2. B.-Y. Chen, On a variational problem of hypersurfaces (mimeograph).

3. K. Shiohama and R. Takagi, A characterization of a standard torus in $E^{3}, \mathrm{~J}$. Differential Geometry 4 (1970), 477-485. MR 43 \#2646.

4. T. J. Willmore, Note on embedded surfaces, An. Şti. Univ. “Al. I. Cuza” Iaşi Secț. I a Mat. 11B (1965), 493-496. MR 34 \#1940.

Department of Mathematics, University of California, los Angeles, CaliFORNIA 90024 